\title{
Brain SPECT imaging in Sydenham's chorea
}

O.G.P. Barsottini ${ }^{1}$

H.B. Ferraz ${ }^{1}$, M.M. Seviliano ${ }^{2}$ and $\mathrm{A}$. Barbieri ${ }^{2}$

\author{
${ }^{1}$ Setor de Distúrbios do M ovimento, Departamento de Neurologia and \\ 2 Setor de Medicina Nuclear, Departamento de Diagnóstico por Imagem, \\ Universidade Federal de São Paulo, São Paulo, SP, Brasil
}

\section{Correspondence \\ H.B. Ferraz \\ Al. Casa Branca, 799, Apto. 72 \\ 01408-001 São Paulo, SP \\ Brasil \\ Fax: +55-11-3064-6585 \\ E-mail: henrique@ neuro.epm.br}

Publication supported by FAPESP.

Received April 6, 2001

Accepted January 28, 2002

\section{Abstract}

The objective of the present study was to determine whether brain single-photon emission computed tomography (SPECT) imaging is capable of detecting perfusional abnormalities. Ten Sydenham's chorea (SC) patients, eight females and two males, 8 to 25 years of age (mean 13.4), with a clinical diagnosis of SC were submitted to brain SPECT imaging. We used HMPAO labeled with technetium-99m at a dose of $740 \mathrm{MBq}$. Six examinations revealed hyperperfusion of the basal ganglia, while the remaining four were normal. The six patients with abnormal results were females and their data were not correlated with severity of symptoms. Patients with abnormal brain SPECT had a more recent onset of symptoms (mean of 49 days) compared to those with normal SPECT (mean of 85 days) but this difference did not reach statistical significance. Brain SPECT can be a helpful method to determine abnormalities of the basal ganglia in SC patients but further studies on a larger number of patients are needed in order to detect the phase of the disease during which the examination is more sensitive.

\section{Key words}

- Sydenham's chorea

- Single-photon emission

computed tomography

- SPECT imaging

- Rheumatic fever

- Brain perfusion

\section{Introduction}

Sydenham's chorea (SC) has been known since the middle ages, but its relationship with rheumatic fever was established only in the 19th century (1). According to Jones' criteria (2), it is currently considered as a major manifestation of rheumatic fever. In $80 \%$ of cases, SC mainly affects children and adolescents, although occurrence in young adults is not rare $(3,4)$.

In 1948 a relationship was established between rheumatic fever and the infection caused by the beta-hemolytic streptococcus of group A (5). There is also evidence that the immune system plays a role in the patho- genesis of the disease by producing antibodies against the subthalamic and caudate nuclei (6). High levels of IgG are found in cerebrospinal fluid during the acute phase of the disease (7).

The number of streptococcal infections is much higher than the number of patients with rheumatic fever. Rheumatic fever, on the other hand, involves a much higher number of patients with clinical abnormalities other than choreic movements. It is currently believed that the presence of streptococcal toxins may induce patient sensitivity and that, depending on genetic and environmental factors, patients may develop the symptoms of rheumatic fever. 
The diagnosis of SC is not easy since the streptococcal infection is difficult to identify, especially because many times there is no association with other manifestations of rheumatic fever. In about 20 to $25 \%$ of the acute chorea cases, no laboratory or clinical evidence of previous rheumatic disease could be found (4). Also, the time between streptococcal infection and the onset of chorea may be long, resulting in negative laboratory tests which might not confirm the "rheumatic state" (4). Thus, the diagnosis of SC is largely presumptive, based merely on the clinical manifestations of chorea in young patients without evidence of other causes.

Some clinical and laboratory data are especially useful to establish the diagnosis of SC. Clinical data include the presence of involuntary movements of insidious onset, absence of a family history of abnormal movements, and absence of drug use before or during the onset of abnormal movements. The laboratory findings include normal thyroid, renal or hepatic function, normal ceruloplasmin levels, negative tests for the rheumatoid factor and antinuclear antibodies, and normal red cell count (8).

Single-photon emission computed tomography (SPECT) depicting regional cerebral perfusion could be a useful method to evaluate basal ganglion function. Preliminary studies show hypoperfusion (hypoconcentration) in degenerative choreas and hyperperfusion in infectious choreas, such as SC $(9,10)$. SPECT was introduced at the beginning of the 80's and has permitted the assessment of regional cerebral perfusion. One of its advantages resides in the low cost of the examination when compared to other techniques of functional analysis of the brain. In SC the number of cases studied by this method is small and conclusions about the value of SPECT in the investigation of patients are not very clear.

Our objective was to determine whether brain SPECT imaging is capable of detecting perfusional abnormalities in a group of pa- tients with the diagnosis of SC and to correlate the clinical findings with the results.

\section{Patients and Methods}

Ten patients were selected from the Outpatients Clinic of the Movement Disorders Unit, Department of Neurology, Federal University of São Paulo, São Paulo, SP, Brazil. There were 8 female and 2 male patients with a mean age of 13.4 years (age range 8 to 25 years).

The study was submitted to and approved by the Ethics Research Committee of São Paulo Hospital, Federal University of São Paulo. Patients or their parents were informed about benefits and risks of the procedure and gave written informed consent to participate in the study.

Inclusion criteria were the presence of choreic movements with onset within the last 6 months, and age from 5 to 25 years.

Exclusion criteria were presence of other neurological manifestations unrelated to chorea, such as cognitive impairment and ataxia; past history of perinatal trauma; family history of abnormal movements, except for SC; presence of thyroid, renal, hepatic or psychiatric diseases preceding or accompanying the abnormal movements; use of drugs known to cause chorea (i.e., neuroleptics, levodopa, phenytoin) preceding or accompanying the abnormal movements, and a history of systemic lupus erythematosus.

Patients did not need to fulfill criteria for the diagnosis of rheumatic fever in order to be included in the study; however, the presence of signs and symptoms of rheumatic fever was decisive for the diagnosis of $\mathrm{SC}$ in case of doubt.

All patients were submitted to clinical examination and to a minimum protocol of laboratory tests which included complete blood count, erythrocyte sedimentation rate, antistreptolysin O, chest X-ray, and electrocardiogram.

Complementary examinations, such as 
thyroid tests, ceruloplasmin or urinary copper were requested only when in doubt about the diagnosis. Brain CT scans were obtained for all patients. Patients suspected to have cardiac disturbances were submitted to an echocardiogram. All patients were seen by a cardiologist regardless of symptoms.

All patients were treated with haloperidol or pimozide, both at initial doses of $1 \mathrm{mg}$ which were gradually increased until symptoms were reduced or produced adverse effects. All patients received prophylactic treatment with benzathine penicillin, 1,200,000 IU every 15 days.

Follow-up consisted of consultations at 3-month intervals over a period of 6 to 24 months and none of the patients had recurrence or chronic manifestation of chorea.

All patients were submitted to brain SPECT imaging at the Nuclear Medicine Unit of the Federal University of São Paulo. HMPAO (Ceretec, Amersham Pharmacia Biotech, Uppsala, Sweden) labeled with technetium-99m (99mTC-HMPAO) was used as the radiotracer (11). All patients had an intravenous access line placed in the arm and remained at rest, with their eyes open, for 30 min in a quiet room with dim light. A dose of $740 \mathrm{MBq}(20 \mathrm{mCi})$ of the radiotracer was injected and image acquisition was started 15 min later. A single-head scintillation camera (Apex SPX-4, Elscint, Haifa, Israel) equipped with a low-energy parallel hole collimator was used. The photopeak was centered at $140 \mathrm{keV}$ with a $20 \%$ symmetric window. Images were acquired with a $64 \mathrm{x}$ 64 matrix without zoom for 360 degrees in a continuous circular orbit. A total of $60 \mathrm{im}$ ages were taken at 6-degree intervals for 20 $\mathrm{s}$ each and a total acquisition time of $20 \mathrm{~min}$. After attenuation correction, images were reconstructed on the transaxial, coronal and sagittal planes and displayed for interpretation. Three nuclear medicine experts, with no knowledge of the clinical status of the patients, interpreted the images visually us- ing the cerebellar activity as reference, and the final conclusion was reached by consensus. Deviations of tracer concentration from normal were graded as mild, moderate and marked.

Data were analyzed statistically by the Mann-Whitney test to compare disease duration between patients with a normal or abnormal SPECT examination, with the level of significance set at $5 \%$.

\section{Results}

We investigated 10 patients, 8 females and 2 males. Table 1 shows the clinical and brain SPECT findings of the patients. None of the patients had a previous streptococcal infection but 5 of them reported a complaint of a recent sore throat (patients Nos. 2, 3, 4, 9 and 10). Association of arthritis and chorea was seen in patients Nos. 1, 4 and 9. Two patients (Nos. 3 and 10) had clinical evidence of carditis. No evidence of abnormal neuropsychiatric features such as obsessivecompulsive symptoms or mood changes was observed in any of the patients. Age ranged from 8 to 25 years (mean \pm SD: $13.4 \pm 4.8$ years). Mean time interval from onset of abnormal movements to consultation was 63.6 days, with a standard deviation of 59.7. All patients presented abnormal movements ranging from mild and only distal choreic movements (such as patient 6) to severe generalized chorea with hypotonia (such as patient 9). All patients had normal brain CT scans.

The cerebral SPECT was abnormal in 6 patients, revealing hyperperfusion of the basal ganglia. All patients with abnormal brain SPECT were females. Patients with abnormal brain SPECT had experienced symptoms for 49 days on average, as compared to 85 days for patients with normal SPECT. This difference was not statistically significant. Figure 1 shows the brain SPECT findings of 2 patients. All patients were on haloperidol or pimozide at the time of the study. 


\section{Discussion}

All patients with abnormal unilateral or bilateral brain SPECT showed hyperconcentration of the radiotracer in the basal ganglia, possibly due to changes in the basal ganglion microcirculation or to a blood-brain barrier abnormality secondary to the infectious process (12). Vasculitis in basal ganglia is observed in SC patients submitted to histologi-

Table 1. Clinical characteristics of Sydenham's chorea patients submitted to brain single-photon emission computed tomography (SPECT).

\begin{tabular}{lcccll}
\hline Patient \# & Gender & $\begin{array}{c}\text { Age } \\
\text { (years) }\end{array}$ & $\begin{array}{c}\text { Duration from } \\
\text { onset to } \\
\text { evaluation (days) }\end{array}$ & Clinical findings & SPECT \\
\hline 1 & F & 12 & 60 & Mild choreic movements R>L, dysarthria & Abnormal* \\
2 & F & 14 & 150 & Mild bilateral choreic movements & Abnormal \\
3 & M & 9 & 60 & Mild bilateral choreic movements & Normal \\
4 & F & 14 & 30 & Moderate bilateral choreic movements & Abnormal \\
5 & F & 12 & 180 & Mild bilateral choreic movements & Normal \\
6 & M & 17 & 90 & Mild unilateral chorea (L) & Normal \\
7 & F & 25 & 20 & Mild choreic movements L>R, dysarthria & Abnormal \\
8 & F & 13 & 6 & Moderate bilateral choreic movements, & Abnormal \\
9 & F & 8 & 10 & $\begin{array}{l}\text { Severe bilateral choreic movements, } \\
\text { hypotonia }\end{array}$ & Normal \\
10 & F & 10 & 30 & Mild bilateral choreic movements & Abnormal \\
\hline
\end{tabular}

*All patients with abnormal SPECT had a hyperconcentration of radiotracer in basal ganglia. L, left; $\mathrm{R}$, right.

Figure 1. Brain single-photon emission computed tomography (SPECT) imaging of patient 2 (abnormal) and patient 3 (normal). The arrowheads in abnormal SPECT (patient 2) indicate the basal ganglia in which an abnormal study presents a hyperconcentration of tracer.
Patient 2: abnormal SPECT
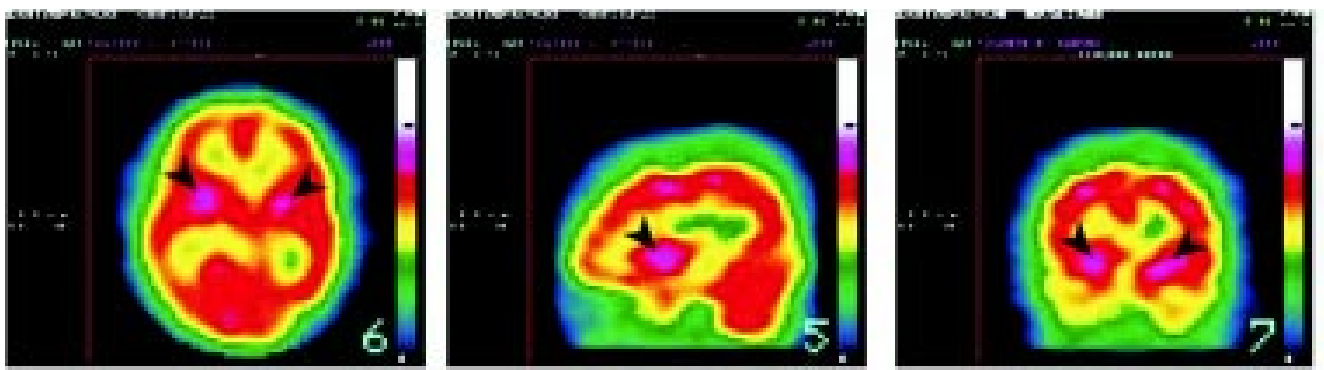

Patient 3: normal SPECT
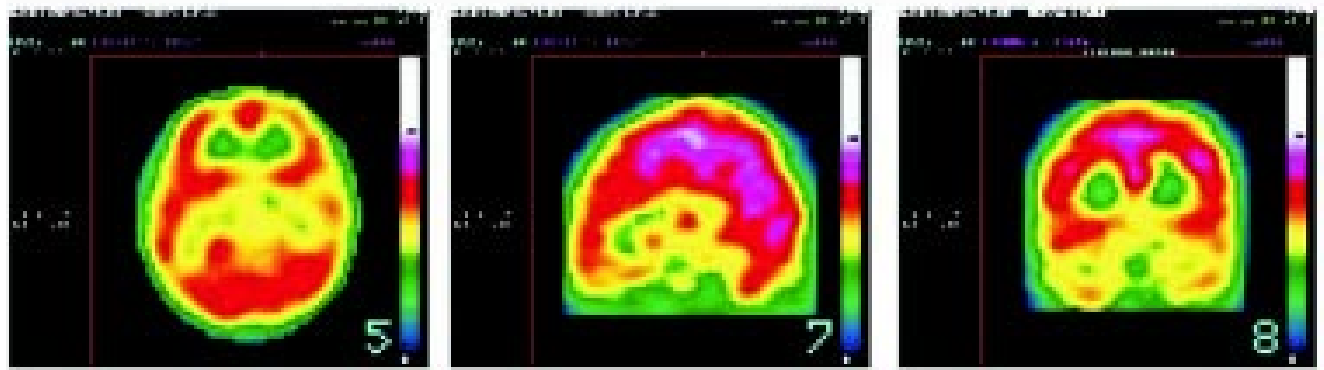
cal postmortem examination (13). The increased radiotracer uptake by basal ganglia could be explained by a blood-brain barrier abnormality induced by the inflammatory process.

In the present study we detected abnormality of the basal ganglia in 6 of 10 patients. This is an expressive number of patients but we cannot explain why 4 patients had a normal brain SPECT despite the presence of SC. Interestingly, we observed that the most symptomatic patients were not necessarily those with an abnormal brain SPECT study. Patient 9, for instance, had severe chorea but her brain SPECT was normal. Perhaps the inflammatory process in the basal ganglia is not directly correlated with the severity of the clinical manifestations. Another explanation for the normal SPECT studies is that the image analysis of the SPECT is based on subjective visual criteria, even though our Nuclear Medicine staff is experienced in the interpretation of these studies. In addition, three experts had to reach a consensus for the final result. An additional explanation is our incomplete understanding of the pathophysiology of SC. For instance, we do not know whether the vasculitis is directly related to the presence of the choreic movements or if it is simply an epiphenomenon (14). Therefore, the patients with normal brain SPECT examinations would just reflect those with chorea but without vasculitis.

All of our 10 patients had clinical abnormalities consistent with the diagnosis of $\mathrm{SC}$, including insidious onset, choreic movements, presence of restlessness, psychological and emotional disturbances and muscular hypotonia (15). We could not establish the correlation between the beta-hemolytic streptococcus and the onset of abnormal movements since in all situations the ASLO examination was within normal limits. An alternative diagnosis such as antiphospholipid syndrome could be proposed for some patients but none of them had evidence of any other disease.

All patients with abnormal brain SPECT (6 patients) were females. Although the reduced number of patients studied does not permit us to conclude that this could apply to all SC patients, female patients seem to have a peculiar clinical evolution of SC as compared to male patients, probably due to hormonal factors (16). We could speculate that these hormonal factors may lead to more prominent inflammatory activity in the basal ganglia and to an abnormal brain SPECT study.

Our group of patients with abnormal brain SPECT averaged 49 days of disease, as opposed to 85 days for patients with normal studies. This difference did not reach statistical significance but perhaps a larger number of patients could confirm this finding. This inverse relationship between duration of the disease and abnormal brain SPECT does not explain patient 2 who had an abnormal test 150 days after the onset of symptoms. We may speculate that disease duration could be one of the factors involved in the abnormality. Other factors, such as recurrent streptococcal infection, might also be involved.

We also know that the brain SPECT abnormality in SC can be reversed (17-19). The normal examination observed in some of our patients could reflect the phase of brain SPECT normalization.

Unfortunately, we did not repeat the brain SPECT on any of our patients. Normalization of the study after disappearance of symptoms would be a demonstration of the specificity and sensitivity of brain SPECT imaging for choreic patients.

In conclusion, we believe that brain SPECT can be a helpful method to identify different phases of SC. A larger number of patients could help to identify the phase of the disease during which brain SPECT imaging is most useful. 


\section{References}

1. Aron AM, Freeman J M \& Carter S (1965). The natural history of Sydenham's chorea: review of the literature and long-term evolution with emphasis on cardiac sequelae. American J ournal of Medicine, 38: 83-95.

2. American Heart Association (1992). Guidelines for the diagnosis of rheumatic fever: J ones criteria, 1992 update. J ournal of the American Medical Association, 268: 2069-2073.

3. Gordon MF (1988). New onset choreiform disorder in an adult with recent group $A$ beta haemolytic streptococcal pharyngitis. J ournal of Neurology, Neurosurgery and Psychiatry, 51: 448-449.

4. Diament A (1972). Valor de alguns exames complementares na Coréia de Sydenham. Arquivos de Neuropsiquiatria, 30: 187214.

5. Scwartzman J, McDonald D \& Perillo L (1948). Sydenham's chorea: report of 140 cases and review of the literature. Archives of Pediatrics and Adolescent Medicine, 65: 6-24.

6. Husby G, Van de Rijn I, Zabriskie J B, Abdin AH \& Williams J r RC (1976). Antibodies reacting with cytoplasm of subthalamic and caudate nucleus neurons in chorea and acute rheumatic fever. J ournal of Experimental Medicine, 144: 1094-1110.
7. Gledhill RF (1986). Selective increase in cerebrospinal fluid immunoglobulin $\mathrm{G}$ in a patient with Sydenham's chorea. J ournal of Neurology, Neurosurgery and Psychiatry, 49: 602-603.

8. Gledhill RF \& Thompson PD (1990). Standard neurodiagnostic tests in Sydenham's chorea [Letter]. J ournal of Neurology, Neurosurgery and Psychiatry, 53: 534535.

9. Kuhl DE, Metter EJ, Riege WH \& Markham CH (1984). Patterns of cerebral glucose utilization in Parkinson's disease and Huntington's disease. Annals of Neurology, 15 (Suppl): S119-S125.

10. Hill A, Herkes GK \& Roche P (1994). SPECT and MRI findings in Sydenham's chorea. J ournal of Neurology, Neurosurgery and Psychiatry, 57: 763.

11. Costa DC, Ell PJ, Cullum ID \& J arritt PH (1986). The in vivo distribution of 99mTcHM-PAO in normal man. Nuclear Medicine Communications, 7: 647-658.

12. Heye $N$, J ergas $M$, Hotzinger $H$, Farahati J , Pohlau D \& Przuntek H (1993). Sydenham's chorea: clinical, EEG, MRI and SPECT findings in early stages of the disease. J ournal of Neurology, 240: 121-123.

13. Colong HS \& Malamud N (1956). Sydenham's chorea, a clinico-pathologic study. Neurology, 6: 672-676.
14. Cardoso F (1998). Infectious and transmissible movement disorders. In: J ankovic J \& Tolosa E (Editors), Parkinson's Disease and Movement Disorders. 3rd edn. Williams \& Wilkins, Baltimore, MD, USA, 945-966.

15. Nausieda PA, Grossman BJ, Koller WC, Weiner WJ \& Klawans HL (1980). Sydenham's chorea: an update. Neurology, 30: 331-334.

16. Cardoso FC, Vargas AP, Oliveira LD, Guerra AA \& Amaral SV (1999). Persistent Sydenham's chorea. Movement Disorders, 14: 805-807.

17. Goldman S, Amrom D, Szliwowski HB \& Detemmerman D (1993). Reversible striatal hypermetabolism in a case of Sydenham's chorea. Movement Disorders, 8: 355-358.

18. Lee PH, Nam HS, Lee KY, Lee BI \& Lee J D (1999). Serial brain SPECT images in a case of Sydenham's chorea. Archives of Neurology, 56: 237-240.

19. Dilenge ME, Shevell MI \& Dinh L (1999). Restricted unilateral Sydenham's chorea: reversible contralateral striatal hypermetabolism demonstrated on single photon emission computed tomographic scanning. J ournal of Child Neurology, 14: 509513. 